\title{
Frontières
}

\section{Les personnes âgées itinérantes - invisibles et exclues. Une analyse de trois stratégies pour contrer l'itinérance}

\author{
Victoria Burns, Amanda Grenier, Jean-Pierre Lavoie, David Rothwell et \\ Tamara Sussman
}

Volume 25, numéro 1, automne 2012

Le vieillissement et sa diversité

URI : https://id.erudit.org/iderudit/1018230ar

DOI : https://doi.org/10.7202/1018230ar

Aller au sommaire du numéro

Éditeur(s)

Université du Québec à Montréal

ISSN

1916-0976 (numérique)

Découvrir la revue

Citer cet article

Burns, V., Grenier, A., Lavoie, J.-P., Rothwell, D. \& Sussman, T. (2012). Les personnes âgées itinérantes - invisibles et exclues. Une analyse de trois stratégies pour contrer l'itinérance. Frontières, 25(1), 31-56.

https://doi.org/10.7202/1018230ar
Résumé de l'article

En se basant sur le concept d'exclusion sociale, cet article soutient que le paradigme de l'État d'investissement social de même que l'accent mis par les politiques sur le vieillissement actif contribuent à l'exclusion des personnes âgées itinérantes (PAI) tant dans les politiques, les pratiques que la recherche. S'appuyant sur trois politiques et plans d'action sur l'itinérance émanant des gouvernements fédéral (canadien), provincial (québécois) et municipal (la Ville de Montréal), cet article montre comment l'exclusion des PAI se manifeste dans ces stratégies. Enfin, cet article démontre le besoin de reconnaître les multiples exclusions des PAI et conclut sur un appel à différents acteurs afin qu'ils adoptent une posture critique face aux discours et aux modèles normatifs qui concourent à l'exclusion de cette population. 


\section{LES PERSONNES ÂGÉES ITINÉRANTES - INVISIBLES ET EXCLUES. UNE ANALYSE DE TROIS STRATÉGIES POUR CONTRER L'ITINÉRANCE}

Victoria Burns
Centre de Recherche et d'Expertise en Gérontologie Sociale (CREGÉS),
C.S.S.S. Cavendish

Amanda Grenier, Ph.D. Centre de Recherche et d'Expertise en Gérontologie Sociale (CREGÉS), C.S.S.S. Cavendish; McMaster University, Department of Health, Aging and Society

Jean-Pierre Lavoie, Ph.D. Université McGill, École de Travail social ; Centre de Recherche et d’Expertise en Gérontologie Sociale (CREGÉS), C.S.S.S. Cavendish; Université du Québec à Montréal, École de Travail social

David Rothwell, Ph.D. Université McGill, École de Travail social

Tamara Sussman, Ph.D. Université McGill, École de Travail social ; Centre de Recherche et d'Expertise en Gérontologie Sociale (CREGÉS), C.S.S.S. Cavendish

\section{RÉSUMÉ}

En se basant sur le concept d'exclusion sociale, cet article soutient que le paradigme de l'État d'investissement social de même que l'accent mis par les politiques sur le vieillissement actif contribuent à l'exclusion des personnes âgées itinérantes (PAI) tant dans les politiques, les pratiques que la recherche. 
S'appuyant sur trois politiques et plans d'action sur l'itinérance émanant des gouvernements fédéral (canadien), provincial (québécois) et municipal (la Ville de Montréal), cet article montre comment l'exclusion des PAI se manifeste dans ces stratégies. Enfin, cet article démontre le besoin de reconnaître les multiples exclusions des PAI et conclut sur un appel à différents acteurs afin qu'ils adoptent une posture critique face aux discours et aux modèles normatifs qui concourent à l'exclusion de cette population.

\section{ABSTRACT}

Using a lens of social exclusion this article contends that the political emphasis on active aging and the social investment state paradigm has reinforced the exclusion of the older homeless people in policy, practice, and research. Drawing on recent Federal (Canadian), Provincial (Quebec), and Municipal responses (City of Montreal) to homelessness, this article demonstrates how the exclusion of older homeless people is perpetuated in these strategies. The article highlights the need to acknowledge the intersecting exclusion of older homeless people and concludes by calling for researchers, policy makers, and practitioners to critically examine discourses and normative models that function to discount this population.

MOTS CLÉS: vieillissement - itinérance - exclusion sociale - politiques publiques - Canada - Québec

KEYWORDS: aging - homelessness - social exclusion - public policies

- Canada - Quebec

Au cours des 30 dernières années, autant l'itinérance que le vieillissement ont retenu l'attention des cercles universitaires et politiques. Toutefois, l'intersection de ces deux «problèmes» sociaux a été largement ignorée par ces milieux. On estime qu'il y a entre 150000 et 300000 personnes itinérantes au Canada (Echenberg et Jensen, 2008). Une étude de Santé Québec (1998) dénombrait quant à elle au-delà de 28000 personnes itinérantes à Montréal, dont près du tiers était âgée 
de 45 ans et plus. Aucune autre étude n'a tenté depuis lors de mesurer l'importance du phénomène à Montréal, la ville de loin la plus touchée par le phénomène de l'itinérance au Québec (idem, 1998). Cela dit, les différents services aux personnes itinérantes de Montréal constatent une augmentation significative du nombre d'usagers (RAPSIM, 2010). Ainsi, de 2008 à 2009, la Old Brewery Mission de Montréal, un des refuges pour personnes itinérantes le plus important du Canada, a vu que le nombre total d'admissions avait augmenté de $34 \%$, soit de 708 à 1077 (OBM, 2009-2010). Alors que le problème de l'itinérance a surtout été abordé sous l'angle du besoin de s'attaquer au nombre croissant de jeunes mendiants (Parazelli, 2002; Bellot, 2001), la «crise» du vieillissement quant à elle s'est construite autour de l'enjeu du coût de la prise en charge d'une population âgée de plus en plus nombreuse vivant à domicile ou en institution (Gee et Gutman, 2000). Ce faisant, on a ignoré le nombre croissant de personnes âgées itinérantes (PAI) (McDonald et al., 2007).

Aucun consensus quant à l'effectif des PAI ne semble exister, les estimations allant de 2 à $27 \%$ de la population itinérante globale (Cohen, 1999). Cet écart s'explique par les différentes définitions de l'itinérance et les multiples seuils utilisés pour classer une personne itinérante comme âgée. Malgré la variabilité des estimations, il est fort probable que la proportion des PAI ira en s'accroissant alors que I'on s'attend à ce que l'effectif de la population âgée soit multiplié par six au cours des prochaines décennies en lien avec l'avancée en âge des baby-boomers (Cohen, 1999), la hausse croissante du coût de la vie, la pénurie de logements sociaux et les compressions dans les programmes sociaux (Laird, 2007). Malgré cette tendance démographique lourde, les PAI sont demeurées pratiquement invisibles.

Cet article débute par la présentation des principaux constats provenant des écrits scientifiques quant aux enjeux spécifiques des PAI. Il examine ensuite de manière critique les paradigmes dominants et les modèles normatifs utilisés dans le champ du vieillissement - mais également dans le champ de l'itinérance - qui mettent de l'avant un discours du vieillissement réussi ${ }^{1}$ (Rowe et Kahn, 1987) ou actif (WHO, 2002) et modulé par les concepts de productivité, d'indépendance et d'autosuffisance qui tendent à blâmer les individus âgés pour leurs difficultés (Grenier, 2012). Enfin, après avoir dressé un bilan de certaines mesures et de programmes destinés aux personnes itinérantes, 
notamment celles qui sont âgées, il procédera à l'analyse de trois stratégies canadiennes pour contrer l'itinérance, (1) la Stratégie des partenariats de lutte contre l'itinérance du gouvernement fédéral (SPLI) (2011-2014), (2) le Plan d'action interministériel en itinérance du Québec (2010-2013) (PAIIQ) et (3) le Plan d'action ciblé en itinérance de la Ville de Montréal (2010-2013) (PAIVM), soulignant I'exclusion des PAI.

\section{CADRE CONCEPTUEL}

Notre analyse est basée sur le concept d'exclusion sociale, un concept incontournable selon nous pour comprendre les expériences des PAI, et le paradigme de l'État d'investissement social. Le terme «exclusion sociale», introduit par l'homme politique français René Lenoir (1974) dans un livre intitulé Les exclus, est adopté par les milieux politiques pendant les années 1980 en Europe (voir Silver, 1994) ainsi qu'au Québec (Poirier et Gagné, 1988). Par exemple en Grande Bretagne le Social Exclusion Unit fut établi en 1997 (Social Exclusion Unit, 2004) et en 2002 une Loi visant à lutter contre la pauvreté et l'exclusion sociale fut introduite au Québec (MESS, 2002). A partir des années 1990, des chercheurs universitaires, français notamment, l'adoptent et en font un concept central dans l'analyse des situations de pauvreté et de marginalisation (Roy et Huturbise, 2007; Noël, 2002; Merrien, 1998; Paugam, 1996; Roy, 1995). Par la suite, plusieurs chercheurs, britanniques notamment, se réapproprient le concept et le transforment afin de répondre aux critiques apportées aux premières formulations de ce concept (Scharf et al., 2005; Vranken, 2002). S'inspirant de ces derniers travaux sur l'exclusion, Billette et Lavoie (2010) définissent l'exclusion comme un processus de non-reconnaissance et de privation de droits et de ressources de certains groupes dans sept dimensions de la vie en société: symbolique, identitaire, économique, liens sociaux, institutionnelle, sociopolitique et territoriale. L'itinérance est le résultat de multiples formes d'exclusion liées à la précarité de l'emploi et aux revenus insuffisants (exclusion économique), aux difficultés d'accès à des logements abordables (exclusion territoriale), aux politiques de désinstitutionalisation mal planifiées (exclusion institutionnelle), à l'absence d'un réseau social de soutien (exclusion des liens sociaux significatifs), et aux préjugés et stéréotypes négatifs (exclusion symbolique). 
Toutefois, notre propos s'intéresse à deux domaines d'exclusion particulièrement pertinentes à l'analyse de l'itinérance, notamment chez les personnes âgées: l'exclusion symbolique et à l'exclusion institutionnelle. La première réfère aux images négatives, aux stéréotypes accolés à un groupe ou encore à son invisibilité. La seconde se définit, quant à elle, comme une absence d'accès aux mesures de protection sociale et sanitaire prévues par les institutions sociales et politiques ou encore par l'absence de contrôle sur les services et les soins reçus (voir Billette et Lavoie, 2010).

L'analyse fait également appel au paradigme politique de l'État d'investissement social, et spécifiquement aux travaux de Jenson et SaintMartin (2003) qui notent l'émergence, dans le contexte de la mondialisation, d'un nouvel État providence qui définit ses politiques en termes d'investissements sociaux. Ce paradigme implique une approche marchande des politiques publiques qui met l'accent sur leur productivité économique - assurer la compétitivité de l'économie nationale - plutôt que sur leur rôle distributif et de maintien du pouvoir d'achat (idem, 2003)2. Dans cette perspective, toujours selon ces auteurs, l'État investit en priorité chez les personnes dont on peut accroître la capacité à contribuer à la productivité de l'économie, l'investissement devant être rentable. Cette finalité l'amène à prioriser les interventions destinées aux mineurs et aux jeunes adultes, risquant fort d'exclure les personnes âgées, notamment les itinérantes, des programmes et des services qu'il développe (exclusion institutionnelle). Par ailleurs, la valorisation par cet État d'investissement social de I'indépendance et de la productivité peut contribuer, quant à elle, à I'exclusion symbolique des PAI.

\section{QUE SAVONS-NOUS DES PERSONNES ÂGÉES ITINÉRANTES?}

La recherche sur les personnes itinérantes âgées est confrontée à deux embûches importantes, soient la définition de l'itinérance et le seuil audelà duquel on considère une personne itinérante «âgée». Au cours des années 1980 et 1990, le gouvernement du Québec s'est graduellement donné une définition de l'itinérance. Voici comment est définie 
une personne itinérante dans le Cadre de référence en itinérance de 2008 du ministère de la Santé et des Services sociaux:

[une personne] qui n'a pas d'adresse fixe, de logement stable, sécuritaire et salubre, à très faible revenu, avec une accessibilité discriminatoire à son égard de la part des services, avec des problèmes de santé physique, de santé mentale, de toxicomanie, de violence familiale ou de désorganisation sociale et dépourvue de groupe d'appartenance stable. (MSSS, 2008, p. 11)

Alors qu'il y a plusieurs plans d'action pour contrer l'itinérance au Canada $^{3}$, qui se donnent chacun une définition de l'itinérance, on ne dispose toujours pas d'une définition qui fait consensus à l'échelle nationale (Echenberg et Jenson, 2008). En fait, définir l'itinérance et une personne itinérante n'est pas une mince tâche, les chercheurs comme les politiques ne s'entendant pas sur «ce qui doit être considéré ou ne doit pas l'être» (Oudshoorn, 2011, p. 2, traduction libre). Il existe de multiples approches pour mesurer le phénomène de l'itinérance, mais, encore une fois, aucune ne fait consensus (Firdion, 2006). Il ne faut dès lors guère se surprendre qu'il n'existe pas un dénombrement fiable des personnes itinérantes au Canada.

Quant à la question du seuil, considérant l'espérance de vie réduite et la prévalence élevée de maladies chez les personnes itinérantes, on tend de plus en plus dans les écrits scientifiques à considérer une personne itinérante comme âgée lorsqu'elle atteint ou dépasse 50 ans (Hecht et Coyle, 2001; Cohen, 1999). Toutefois, aucun consensus n'existe entre les planificateurs, les intervenants et les chercheurs quant à ce seuil. Cela contribue à expliquer, comme nous I'avons mentionné précédemment, pourquoi les estimations de la proportion de PAI parmi la population itinérante varient autant (de 2 à $27 \%$ ). Ce manque de consensus sur leur nombre et, plus généralement, de connaissances sur les PAI a sans doute contribué au manque de reconnaissance, voire I'invisibilité, dont elles sont victimes dans les politiques publiques et les services institutionnels.

Malgré ces embûches, certaines indications laissent entendre que I'on assiste à une extension et une diversification du phénomène de l'itinérance. Roy et Huturbise (2007) notent:

Alors qu'à la fin des années 1980 l'itinérance était principalement le fait des hommes, aujourd'hui on voit des femmes (Roy et al., 2002) 
et des jeunes (Parazelli, 2002; Hurtubise et Laaroussi, 2000) qui vivent cette situation et on voit aussi apparaitre le phénomène des familles itinérantes et celui de la hausse du nombre d'itinérants parmi les populations autochtones (Bellot, 2001) (p.11).

L'augmentation du nombre de femmes itinérantes pourrait indiquer une augmentation du nombre des PAI. En effet, dans une étude réalisée à Toronto auprès de personnes itinérantes chroniques et récentes, McDonald et ses collègues (2007) observent que plus de femmes que d'hommes deviennent itinérantes pour la première fois à un âge avancé. Cohen et Crane (1996) font la même constatation dans leur étude menée à Londres et New York. Les taux de prévalence de l'itinérance chez les femmes âgées auraient considérablement augmenté dans les dernières décennies, laissant ainsi entendre qu'il y aurait aussi une augmentation du nombre de PAI «récentes» (McDonald et al., 2007; Kutza et Keigher, 1991).

Malgré le faible nombre d'études réalisées auprès des PAI, déjà nous pouvons constater que ce groupe est hétérogène. Ainsi, quatre importants sous-groupes de PAI ont été identifiés: les chroniques, les récents, les «à risque», et les invisibles. Les personnes âgées dites chroniques sont celles qui ont été sans domicile fixe la plus grande partie de leur vie adulte et qui sont désormais rendues à un âge avancé (Cohen et Sokolovsky, 1989). Ce sous-groupe, probablement le plus visible des quatre, évoque les images stéréotypées du vagabond qui aurait en quelque sorte «choisi» ce mode de vie (McDonald et al., 2007). Le sous-groupe des itinérants «récents» comprend les personnes qui deviennent itinérantes pour la première fois à un âge avancé (ibid., 2007), ce groupe compte de nombreuses femmes. Les personnes «à risque» d'itinérance sont celles qui vivent une situation de logement précaire et qui sont susceptibles de perdre leur logement à la suite d'une reprise ou la fin du bail sans être en mesure d'envisager d'autres options (Morris et al., 2005). Un quatrième sous-groupe, qualifié $d^{\prime}$ «invisible», est composé de personnes sans domicile personnel et qui demeurent chez des amis ou des membres de la parenté (couch surfing). Sans ce logement temporaire, ces personnes vivraient dans la rue ou dans un refuge (idem, 2005). Toutefois, compte tenu de la nature cyclique de l'itinérance, les chroniques, les récents, et les «à risque» peuvent, à un moment donné dans leur trajectoire d'itinérance, faire partie du sous-groupe des «invisibles». Les trois derniers 
sous-groupes sont très peu connus, n'ayant fait l'objet de recherche que très récemment (McDonald et al., 2007). Notons également que des recherches ont porté sur d'autres sous-groupes spécifiques de PAI tels que les vétérans (Applewhite, 1997), les autochtones (Kramer et Barker, 1996), les personnes en besoin de soins palliatifs (Cagle, 2009) ainsi que les immigrants (McDonald et al., 2007). Ces études démontrent que le croisement de certains positionnements sociaux influence tant le risque que l'expérience de l'itinérance. Malgré le progrès que constitue cette reconnaissance de l'hétérogénéité des membres de la population itinérante âgée, de leurs trajectoires et de leurs besoins, l'image dominante dans les milieux politiques et institutionnels et dans le public demeure celle de l'itinérance liée à des pathologies personnelles ou d'un choix de style de vie en marge de la société (Parsell et Parsell, 2012; Belcher et al., 2005). Cette image peut à son tour conforter celle des PAI comme étant essentiellement de jeunes itinérants par «choix» et qui ont vieilli et pour lesquels on peut faire peu de choses. La pratique semble bien indiquer que les PAI sont plus nombreuses que nous serions amenés à le penser, car elles se retrouvent dans les quatre groupes identifiés précédemment et non chez les seules chroniques.

\section{L'EXCLUSION SYMBOLIQUE ET INSTITUTIONNELLE DES PAI}

\section{- Une double étiquette: vieux et itinérant}

Valoriser l'indépendance, la productivité et l'autosuffisance contribue à perpétuer l'exclusion symbolique des personnes âgées, a fortiori des PAI, car ces groupes se voient étiquetés négativement comme dépendants (Grenier, 2012, 2007, 2003). Les concepts associés d'indépendance et d'autosuffisance revêtent un caractère normatif, renforcé d'ailleurs par l'État d'investissement social, la dépendance physique ou financière ne pouvant alors qu'être perçue comme indésirable, même si cette dépendance est également le produit des programmes de I'État ${ }^{4}$. Il s'ensuit que les groupes qui se voient accoler ces images de dépendance sont rapidement perçus comme des fardeaux financiers et sociaux pour la société à laquelle ils ne contribuent guère, toujours 
selon cette perception. C'est le cas des personnes âgées définies comme fragiles au plan physique (Grenier, 2007) et les personnes itinérantes vues comme dépendantes financièrement. Par ailleurs, I'itinérance est largement décrite, tant dans les politiques (ex.: SPLI) que par les milieux médicaux et le grand public, comme un déficit personnel, souvent lié à des problèmes de santé mentale ou de toxicomanie (Belcher et al., 2005). Pourtant, de nombreuses recherches sociales démontrent que l'itinérance est le résultat d'une combinaison de facteurs certes individuels, tels que les maladies mentales ou physiques ou l'alcoolisme (Crane, 2001), mais également de facteurs structurels, tels que la perte des prestations d'aide sociale, l'absence de logements abordables ou l'augmentation des loyers, les politiques de désinstitutionalisation et les reconfigurations familiales (McDonald et al., 2007; Roy et Huturbise, 2007; Laberge et al.,1998). Les réponses politiques en matière d'itinérance que véhiculent ces représentations ont pour effet de faire porter aux individus le blâme pour leur situation de dépendance, contribuant ainsi à l'exclusion symbolique des personnes âgées, des personnes itinérantes et, a fortiori, les PAI. Évidemment, cette perception fausse évite de souligner les barrières structurelles au «vieillissement réussi», notamment les situations de pauvreté prolongées ou encore l'insuffisance et l'inadéquation des services sociaux et de santé.

Par ailleurs, pour l'État d'investissement social, les personnes âgées sont difficilement considérées comme un investissement puisque l'on ne s'attend pas à ce qu'elles intègrent la force de travail (Jenson et Saint-Martin, 2003). En fait, comme nous le verrons, de nombreuses mesures de lutte à l'itinérance visent à stabiliser les personnes afin qu'elles puissent dénicher un emploi et ainsi devenir des citoyens productifs et méritants. On peut penser que ces mesures se révèlent moins pertinentes pour les personnes suffisamment âgées - les 65 ans et plus - pour lesquelles on ne s'attend généralement pas qu'elles intègrent le marché du travail (ibid., 2003). Dans un tel contexte, il est peu surprenant que les politiques, mais aussi la recherche, aient ciblé en priorité les jeunes et les personnes en âge de travailler qui, s'ils sont soutenus et logés adéquatement, pourraient occuper un emploi et devenir des citoyens productifs et autosuffisants. 


\section{Les services sociaux lacunaires pour les PAI}

Les personnes itinérantes, peu importe leur âge, sont confrontées à de multiples barrières qui limitent leur accès aux services, notamment les services de santé (Roy et Huturbise, 2008; Denoncourt et al., 2000). Une fois qu'elles y accèdent, elles sont souvent confrontées à des services normatifs, stigmatisants et discriminants (Roy et Hurtubise, 2008). Selon ces auteurs, cette situation s'expliquerait entre autres par les préjugés et le manque de formation des intervenants. Cette situation d'exclusion serait toutefois encore plus marquée chez les PAI. En effet, Quirion et Di Gennaro (2000) notent que les personnes présentant de multiples problèmes subissent davantage d'exclusion avec le cloisonnement des disciplines et des services qui ne sont alors pas en mesure de recevoir cette clientèle complexe et hors norme, qui a pourtant d'importants besoins de reconnaissance. Cela risque d'être souvent le cas des personnes qui sont à la fois itinérantes et âgées.

Les difficultés d'accès aux services sociaux destinés aux personnes âgées tels que 1) les pensions de vieillesse, 2) les soutiens au logement, et 3) les autres bénéfices liés à l'âge peuvent contribuer à l'augmentation de la prévalence de l'itinérance au troisième âge (Means, 2007; Pannell et al., 2002; Hecht et Coyle, 2001). De plus, plusieurs PAI n'ont pas l'âge requis pour bénéficier de ces mesures. Par exemple, Hecht et Coyle (2001) rapportent que seulement le quart des 262 participants âgés de 55 ans et plus résidant dans un refuge californien reçoivent de I'aide sociale. Au Canada, il faut être âgé d'au moins 65 ans pour percevoir une pension de la Sécurité de la vieillesse éventuellement 67 ans avec la réforme prévue par le gouvernement canadien - ce qui prolonge la période de vulnérabilité chez les PAI (Montpetit, 2012). Au Québec, même si l'âge d'éligibilité diminuera d'un an à chaque année d'ici 2015, le seuil d'éligibilité pour l'allocation logement est présentement de 54 ans (Revenu Québec, 2011). Ces seuils rigides fondés sur l'âge font en sorte que des personnes incapables de travailler sont trop jeunes pour bénéficier de ces mesures.

Les PAI risquent également de connaître des problèmes d'accessibilité aux programmes généraux pour personnes âgées par manque d'adresse fixe. Nous voyons mal par exemple comment les PAI pourraient recevoir des services tels que les services à domicile. Les effets d'une dépendance à long terme d'une substance sont à même de créer une 
barrière importante à l'accès aux services de santé et sociaux prévus pour les aînés selon Pannell (2005). Après avoir vécu de longues années en marge de la société, plusieurs PAI pourraient refuser d'intégrer certaines activités sociales traditionnelles pour aînés (ex.: club d'Âge d'or), ce qui pourrait renforcer leur isolement social (Homeless Link, août 2009). En revanche, même si une PAI désirait recevoir un service de santé, le resserrement continuel des critères d'admissibilité aux programmes et services de santé peut faire en sorte que la personne «n'est pas assez intoxiqué(e), assez malade ou trop autonome» pour y accéder (Roy et Huturbise, 2008, p. 18).

\section{- Les services de logement pour les PAI, un problème d'accès}

Dans les 30 dernières années au Canada et au Québec, plusieurs écrits ont établi un lien entre la pénurie de logements sociaux et l'accroissement de l'itinérance (Layton, 2008). Pour ce qui est des personnes âgées, Gascon et Olazabal (2011) soulignent qu'à Montréal, «le logement abordable pour aînés souffre d'un déficit de place» (p. 137). Présentement, I'Office municipal d'habitation de Montréal (OMHM) offre aux personnes âgées de 60 ans et plus 1259 logements abordables avec services (OMHM, 2011) - dont sont exclus les PAI de 50 à 59 ans. À I'international, on remarque en Grande-Bretagne que le manque de places serait particulièrement problématique pour les personnes âgées car elles ont davantage de difficulté à trouver un logement abordable, les services ayant tendance à donner la priorité aux familles avec enfants plutôt qu'aux aînés (Crane et Warnes, 2007).

\section{Les refuges et les services d'habitation de dernier recours}

Les PAI rencontrent également des difficultés quant aux services d'hébergement en urgence. D'abord, la hausse constante du nombre d'utilisateurs des trois plus grands refuges à Montréal, où les taux d'occupation approchent ou même dépassent les $100 \%$, font qu'ils doivent de plus en plus souvent refuser des personnes itinérantes (voir RAPSIM, 25 novembre, 2011). Par ailleurs, les PAI éviteraient les 
refuges car elles y seraient souvent victimes d'abus (Grenier, 1996). Ensuite, le manque de ressources en logement social aurait davantage de conséquences pour les personnes âgées car, comparativement aux personnes itinérantes plus jeunes, les PAI sont à risque de tomber dans un cycle d'itinérance chronique si elles ne sont pas relogées rapidement (Crane et Warnes, 2007).

\section{- Les défis du relogement des personnes âgées itinérantes}

Il y a actuellement une tendance dans les politiques sur l'itinérance surtout au Royaume-Uni et aux États-Unis, plus récemment au Canada - à favoriser le relogement des personnes itinérantes plutôt que de se limiter à la seule mise en place de services d'urgence. Un modèle relativement récent et original de politique de transition hors de l'itinérance est celui du Housing First (HF) ou «logement d'abord». Introduit dans les années 1990 dans la ville de New York avec le projet Pathways to Housing (Tsemberis et Eisenberg, 2000), en 2005 à Toronto avec Streets to Home (Falvo, 2009) et en 2009 à Montréal avec le Projet Chez Soi (Commission de la Santé Mentale, 2012), ce modèle prône le déplacement rapide de la personne ou du ménage itinérant de la rue à son propre logement permanent sans prévoir d'interventions préalables au relogement ${ }^{5}$. Ancrée dans un climat socioéconomique qui valorise l'indépendance et la productivité, cette approche du HF suppose que déplacer la personne itinérante directement de la rue ou du refuge à un appartement conventionnel lui permettra de devenir auto-suffisante, indépendante, ou de le redevenir, et, éventuellement, de se réinsérer dans le marché du travail (Klodawsky, 2009). Les taux de réussite du modèle HF sont quasi spectaculaires: entre 85 et 90 pour cent des participants au programme sont toujours hébergés lors du suivi cinq ans plus tard (Chez Soi Rapport Intérimaire, 2012; Tsemberis et Eisenberg, 2000). Le modèle HF serait beaucoup plus économique et efficace que le modèle traditionnel de Continuum of Care (CC) (Klodawsky, 2009). Toutefois, cette approche aurait été développée dans un contexte néolibéral (idem, 2009) qui priorise les adultes en âge de travailler et qui, par ailleurs, pourrait être plus efficace auprès des populations itinérantes plus jeunes. 
Peu d'études ont analysé les taux et les facteurs de réussite du relogement chez les PAI. Crane et Warnes (2007) ont mené I'une des rares études ethnographiques et longitudinales sur le relogement (il n'est pas spécifié dans cette étude si le relogement suit le modèle HF). Des 64 PAI qui ont participé à ce programme de relogement britannique, seulement $44 \%$ d'entre elles étaient toujours logées après deux ans. Ces auteurs notent que les difficultés soulevées par le manque d'autonomie et l'instabilité des PAI et l'absence de soutiens spécialisés pour répondre à ces difficultés expliquent ce taux plus faible de réussite. Dans la même veine, Hecht et Coyle (2001) soulignent que la probabilité de réussite d'une transition des PAI vers une vie autonome est faible, car il existe peu de services de soutien.

Le relogement des PAI requérant des soins rencontre également un obstacle supplémentaire : elles sont souvent exclues des services gérontologiques à cause de leur consommation d'alcool ou de leurs problèmes de comportement (Crane et Warnes, 2007). Vivre de manière autonome dans un logement conventionnel serait donc possible pour certaines personnes âgées selon ces auteurs, mais encore faut-il qu'elles reçoivent des soins supplémentaires et un soutien sur place, ce qui est peu fréquent dans les modèles actuels de relogement à long terme.

\section{TROIS STRATÉGIES POLITIQUES POUR CONTRER L'ITINÉRANCE}

Au cours des dernières années, l'augmentation du nombre de personnes itinérantes au Canada a inquiété les politiciens. Il en est résulté de multiples plans d'action et de nombreuses promesses de la part de partis politiques. Cette réaction a été alimentée par des soucis au plan de la sécurité publique soulevés par ces jeunes mendiants ou ces squeegees ${ }^{6}$ qui occupent l'espace public et par une volonté de les réinsérer rapidement au marché du travail (Parnaby, 2003). Ces représentations semblent sous-tendre les trois politiques canadiennes qui retiennent notre attention: la Stratégie des partenariats de lutte contre l'itinérance (SPLI) du gouvernement canadien (2011-2014), le Plan d'action interministériel en itinérance du Québec (PAIIQ) (2010-2013) et Agir résolument pour contrer l'itinérance: Plan d'action ciblé en itinérance (2010-2013) de la Ville de Montréal 
(PAIVM). Nous avons choisi d'analyser ces trois politiques en particulier parce qu'elles sont actuellement en cours et représentent les trois niveaux gouvernementaux du Canada (national, provincial et municipal). Nous nous sommes intéressés à ces trois stratégies à cause des messages différents qu'elles véhiculent au sujet de l'itinérance et du vieillissement.

\section{- La Stratégie des partenariats de lutte contre I'itinérance (SPLI) (2011-2014)}

La SPLI a été lancée en 2007 sous le gouvernement conservateur par Ressources humaines et développement des compétences Canada (RHDCC, 2011). Avec un budget annuel de 135 millions de dollars par année jusqu'en $2014^{7}$, le but de la SPLI est de prévenir et de réduire I'itinérance en soutenant et finançant différents programmes communautaires dans 61 communautés désignées au Canada (idem, 2011). Malgré la réalisation de plusieurs projets communautaires ${ }^{8}$, le développement de logements et la publication d'études fortes intéressantes, cette politique semble avoir eu, somme toute, peu d'effets quant à l'éradication de l'itinérance quand on considère le nombre croissant d'itinérants. Au contraire, son approche à la pièce, son coût élevé, son inefficacité et le caractère ponctuel et limité de ses subventions ont soulevé de sérieuses critiques (Davies, 2010 ; Hulchanski, 2009 ; RSIQ, 2009). Toutefois, au-delà de son coût élevé et des problèmes d'implantation, le SPLI est sujet à caution à cause de son idéologie sous-jacente.

Ainsi, sur son site web en version anglaise sous la rubrique «Understanding Homelessness», on peut y lire: «In Canada, homelessness has a thousand faces. The reasons people are homeless in our country are many and varied. Many of the homeless have become disconnected from their families and communities. There are also those who have not been able to find or keep jobs » (RHDCC, 2011) $)^{9}$. En soutenant que I'itinérance est une question de relations interpersonnelles difficiles et de personnes incapables de trouver ou de conserver un emploi, le présupposé sous-jacent est que l'itinérance est due à des défaillances personnelles ou relationnelles. Le nombre insuffisant d'emplois ou les problèmes plus larges du marché du travail n'occupent alors qu'une place marginale dans la question de l'itinérance. 
L'insistance sur le retour au travail est aussi évident en considérant qu'une des priorités de la Stratégie est de mettre «l'accent sur I'intégration au marché du travail, lorsque cela sera possible, afin de favoriser la stabilité économique des sans abri et des personnes à risque de le devenir» (RHDCC, 2011). De plus, la SPLI vise à mettre «en place des structures et des mesures de soutien pour aider les sans-abri et les personnes à risque de le devenir à jouir d'une autonomie et à participer pleinement à la société». Ce discours valorisant la participation au marché du travail, l'autosuffisance, I'intégration au marché et la stabilité économique fait l'impasse sur la réalité des PAI qui, comme nous l'avons indiqué, n'ont que très peu de chances d'intégrer le marché du travail à cause de leur double handicap: leur passé d'itinérant et leur âge. En effet, la SPLI reprend le discours dominant qui présente l'itinérance comme résultant de problèmes personnels ou d'un choix plutôt que de facteurs structurels tels que la pauvreté et les inégalités, malgré les recherche qui démontrent l'importance de ses facteurs (Murphy, 2009; Roy et Hurtubise, 2007; Poirier et al., 1999). En insistant sur la réinsertion dans le marché du travail, le discours de la SPLI est discriminant à l'égard des adultes âgés en ne tenant pas compte de leurs capacités. De plus, malgré la reconnaissance que l'itinérance peut toucher les personnes de tout âge, la SPLI en ciblant la responsabilité individuelle et le travail productif met de l'avant un discours d'exclusion qui ne répond aucunement aux besoins particuliers des PAI.

\section{- Le Plan d'action interministériel en itinérance du Québec (PAIIQ) (2010-2013)}

Échelonné sur trois ans, ce plan provincial associe dix ministères et organismes gouvernementaux et s'articule autour de cinq priorités: la prévention, la stabilisation résidentielle, la coordination des interventions, la cohabitation dans l'espace public et l'accroissement de la recherche. Toutefois, le discours sur l'intégration au marché du travail semble également dominant dans le PAIIQ. En effet, c'est l'emploi qui semble ressortir comme priorité. Ainsi, le mot «emploi» est utilisé 27 fois dans les 52 pages que comporte le Plan d'action. Contrairement à la SPLI cependant, le PAIIQ fait explicitement référence aux besoins particuliers des PAI dans la section intitulée «Points marquants pour 
orienter l'action». On peut y lire: «La grande vulnérabilité des personnes âgées en situation d'itinérance demande que des services convenant à leurs besoins soient mis de l'avant, notamment au regard du logement et des soins de santé adaptés» (PAIIQ, 2009, p. 20). Cette recommandation repose cependant sur une problématisation sommaire de la situation des PAI, se limitant à constater leur longue durée de séjour en ressources d'hébergement et leur grande vulnérabilité. Par ailleurs, au plan de la recherche, le PAIIQ propose en priorité d'accroître la recherche «chez les immigrants, notamment les jeunes» et «chez les autochtones en milieu urbain» (p. 42), laissant pour compte les PAI qui ont pourtant fait l'objet de peu d'études spécifiques au Québec.

Le PAIIQ est critiqué par le Réseau d'aide aux personnes seules et itinérantes de Montréal (RAPSIM) ${ }^{10}$ et le Réseau Solidarité Itinérance du Québec (RSIQ) ${ }^{11}$ (voir Corriveau, 2011) qui constatent que plusieurs mesures telles que l'amélioration de l'accès au logement ne sont pas respectées. En fait, le RAPSIM soutient «que les mesures demeurent floues quant aux moyens prévus pour les mettre en œuvre...et que certaines mesures ne proposaient en réalité que le statu quo» (25 novembre, 2011, p.2). Ce bilan du PAIIQ semble également s'appliquer à la mesure énoncée en faveur des PAI. Nos consultations avec des intervenants qui travaillent auprès des PAI indiquent que c'est effectivement plutôt le statu quo en ce qui concerne cette mesure, les services destinés aux PAI demeurant tout à fait insuffisants alors que le nombre de personnes itinérantes âgées ne cesserait de croître.

Signalons enfin que le gouvernement du Québec annonçait en octobre 2012 son intention d'adopter une politique en itinérance au Québec (RAPSIM, 2012). Cette intention saluée par le RSIQ permettra-t-elle une plus grande reconnaissance des besoins spécifiques des PAI et la mise en place de services spécifiques à leur situation? Notons pour le moment que la plateforme actuelle de revendication du RSIQ, composée de 94 actions regroupées en six axes (droit de cité, droit au logement, droit à un revenu décent, droit à la santé, droit à l'éducation, droit à un réseau d'aide et de solidarité) et s'adressant à tous les paliers de gouvernement, ne cible aucunement les PAI (voir les 94 actions dans le Plateforme de revendications RSIQ, 2006). Malgré 
la reconnaissance dans la Plateforme que l'itinérance se vit à tous les âges, l'absence de mesures ciblées répondant à la spécificité de la situation des PAI risque de perpétuer I'invisibilité dans laquelle se trouve cette population.

\section{- Le Plan d'action ciblé en itinérance de la Ville de Montréal (PAIVM) (2010-2013)}

Le plan énonce 11 actions prioritaires allant de la construction de nouveaux logements sociaux à la consolidation d'une unité policière mobile d'intervention en itinérance en passant par I'amélioration du partage de l'espace public (Ville de Montréal, 2010). Une des principales interventions suggérées pour réduire les problèmes de cohabitation dans l'espace urbain est d'encourager la réinsertion à l'emploi pour les personnes hors du marché du travail:

La Ville considère également avec beaucoup d'intérêt le développement de projets d'employabilité visant l'embauche de personnes en situation d'itinérance ou à risque, qui ont été initiés par certaines sociétés de développement commercial. Des ententes, établies conjointement avec les organismes communautaires travaillant auprès des personnes en situation d'itinérance, offrent une opportunité d'emploi et de réinsertion sociale à des personnes éloignées du marché du travail (p.12).

Tout comme le SPLI, le PAIVM reprend le discours de l'autosuffisance et vise la réinsertion au marché de travail. Tel que mentionné, ce discours et cette approche excluent la plupart des PAI. Contrairement au plan d'action provincial qui reconnaît la spécificité des PAI, il n'y a aucune référence aux PAI dans le PAIVM qui pourtant vise à assurer des services à des populations très spécifiques telles les femmes et les Inuits. Cette omission est d'autant plus surprenante que l'on s'attend à ce qu'une personne sur cinq à Montréal en 2026 soit âgée de 65 ans et plus, ce qui devrait se répercuter sur l'effectif de la population âgée itinérante (Agence de la santé et des services sociaux de Montréal, 2008). Malgré cette tendance, les PAI sont tout à fait invisibles dans ce plan d'action. 


\section{QUE FAIRE MAINTENANT?}

Les PAI se situent à l'intersection des enjeux soulevés à la fois par I'itinérance et le vieillissement (voir Negron-Poblete, 2012; Roy et Huturbise, 2007). Au plan de l'itinérance, les trois politiques ou plan d'action analysés n'abordent pas les mêmes dimensions et n'arrivent pas aux mêmes conclusions. Ces trois visions différentes illustrent aussi la nature fragmentée du dossier de l'itinérance au Canada ainsi qu'au Québec. Une des premières questions est de savoir quel niveau gouvernemental devrait être le principal maître d'œuvre dans le dossier de l'itinérance? Le fédéral, le provincial ou le municipal? Quel rôle doit être dévolu au milieu communautaire? Est-ce le temps d'introduire une politique «globale» d'itinérance telle que proposée depuis 2006 par le RSIQ? Quant à la spécificité des personnes âgées, la question de la place faite aux PAI dans les politiques et les programmes se pose. Malgré la mention qui est faite d'elles dans le PAIIQ, elles demeurent négligées, voire invisibles. Si elles présentent des enjeux et des besoins similaires à l'ensemble des personnes itinérantes, elles nous semblent présenter des spécificités qui militent en faveur d'une reconnaissance de cette spécificité. Il faudrait sans doute s'inspirer de I'exemple britannique où la UK Coalition on Older Homeless (2007) a pour mandat de développer des services spécialisés pour les PAI et fait la promotion de la mise sur pied d'activités de jour structurées afin de faciliter leur transition vers des logements permanents. Il ne faut toutefois pas oublier le risque que la mise en place de services spécifiques puissent constituer des ghettos qui à long terme pourraient se révéler dommageables.

Afin d'assurer la mise en place de services répondant aux besoins des PAI, notamment au regard du logement et des soins de santé, de nombreuses questions demeurées en suspens doivent être répondues. Par exemple, en ce qui concerne le relogement des PAI, quel modèle parmi ceux développés - Housing First, Continuum of Care - serait le mieux adapté à cette population? Une combinaison des deux? D'autres approches seraient-elles possibles? Comment adapter à cette population les modèles dominants de services de santé aux personnes âgées, nous pensons notamment aux services de longue durée centrés sur le domicile et l'institution? Compte tenu du nombre croissant des PAI, le besoin de recherche et de politiques ou de pratiques innovantes apparaît évident. 
Un autre ordre de questions se pose également. En effet, comment expliquer la persistance d'un discours qui présente l'itinérance comme un «choix de vie» ou comme étant essentiellement une répercussion de failles ou de problèmes personnels malgré les acquis de la recherche? Comment lutter contre le maintien des modèles politiques néolibéraux dominants en matière d'itinérance, qui mettent de l'avant la productivité, l'autosuffisance et la responsabilité individuelle? Ces modèles et les pratiques qui en découlent contribuent à l'exclusion des personnes vivant à la marge de la société, notamment les PAI. Qu'est-ce qui rendraient alors les politiques publiques plus justes pour les groupes marginaux tels que les PAI? Quels sont les principaux défis et les contradictions qui nous guettent? Nous pensons ici au dilemme entre le besoin de mesures adaptées ou spécifiques et le risque de créer des ghettos de PAI. Toutes ces questions méritent notre attention afin de déconstruire les présupposés concernant l'intersection du vieillissement et de l'itinérance.

Le but de cet article n'est pas de proposer des solutions - nous n'en sommes pas encore là - mais plutôt d'enclencher une réflexion et une critique des discours dominants et des modèles normatifs de services qui contribuent à l'exclusion des PAI. L'invisibilité des PAI ou la non-reconnaissance de leurs besoins et de leur spécificité est le reflet d'un enjeu structurel plus large qui fait en sorte que les personnes âgées et divers sous-groupes de cette population ne sont pas reconnus (Charpentier et al., 2010). Le concept d'exclusion sociale permet de questionner les structures sociales et $d^{\prime}$ 'identifier les failles de notre société tout en fournissant des pistes d'action possibles pour y pallier (Billette et Lavoie, 2010). Ceci n'est que le début d'une discussion car les études sur les PAI demeurent trop peu nombreuses. Il faudrait par ailleurs davantage de recherches adoptant une approche de gérontologie critique ${ }^{12}$ (Grenier, 2012, Estes et al., 2003) afin d'étudier l'invisibilité des PAI dans les plans d'actions et les politiques consacrés à l'itinérance, les modèles normatifs de transition dans les parcours de vie et les processus de stratification sociale qui sous-tendent la marginalisation.

En soulignant les difficultés rencontrées dans la recherche, les politiques et I'intervention menées sur l'intersection du vieillissement et de l'itinérance, nous espérons amener les chercheurs, les planificateurs et les intervenants à reconnaître les présupposés problématiques, à 
remettre en question les discours dominants qui orientent leur travail et à proposer des voies d'action concrètes pour cette population qui demeure invisible et exclue socialement. À cet effet, il nous semble important de rappeler que l'inclusion n'est pas un enjeu personnel mais bien collectif (Billette et Lavoie, 2010). Il y a une place à faire dans la société pour les personnes marginalisées, ce qui inclut les PAI.

\section{BIBLIOGRAPHIE}

ALBERTA SECRETARIAT FOR ACTION ON HOMELESSNESS (2008). «A plan for Alberta Ending homelessness in 10 years», Edmonton, Alberta Secretariat for action on Homelessness, $44 \mathrm{p}$.

APPLEWHITE, S. L. (1997). «Homeless Veterans: Perspectives on Social Service Use», Social Work, vol. 42, n 1, p. 19-30.

BELCHER, J. R., B.R., DEFORGE et D.A. ZANIS (2005). «Why has the social work profession lost sight of how to end homelessness?», Journal of Progressive Human Services, vol. 16, no 2, p. 5-23. doi: 10.1300/ J059v16n02_02

BELLOT, C. (2001). Le monde social de la rue: expériences des jeunes et pratique d'intervention à Montréal, thèse de doctorat dirigée par MarieMarthe Cousineau, Montréal, Université de Montréal, 290 p.

BILLETTE, V. et J.-P. LAVOIE (2010). «Introduction. Vieillissements, exclusions sociales et solidarités», dans M. Charpentier et al. (dir.), Vieillir au pluriel. Perspective sociales, Québec, Presses de I'Université de Québec, p. 1-22.

CAGLE, J. G. (2009). «Weathering the storm: Palliative care and elderly homeless persons», Journal of Housing for the Elderly, vol. 23, no 1/2, p. 29-46.

CHARPENTIER, M., N. GUBERMAN, V. BILLETTE, J.-P. LAVOIE, A. GRENIER et I. OLAZABAL (2010). Vieillir au pluriel. Perspective sociales, Québec, Presses de I'Université de Québec, 496 p.

CITY OF VANCOUVER (2005). Homeless Action Plan, Vancouver, City of Vancouver Housing Centre, 66 p.

COHEN, C. et J. SOKOLOVSKY (1989). Old men of the Bowery: Strategies for survival among the homeless, New York, Guilford, 248 p.

COHEN, C. (1999). «Aging and homelessness», The Gerontologist, vol. 39, $\mathrm{n}^{\circ} 1$, p. 5-15.

COHEN, C. et M. CRANE (1996). «Old and homeless in London and New York City: A cross-national comparison», dans D. Bhurga (dir.), Homelessness and Mental Health, London, Cambridge University Press, 344 p.

COMMISSION DE LA SANTÉ MENTALE (septembre, 2012). «At Home/ Chez Soi. Rapport intérim», <http://www.mentalhealthcommission.ca/ SiteCollectionDocuments/AtHome-ChezSoi/AtHome_InterimReport_ENG. pdf>, consulté le $1^{\text {er }}$ octobre 2012.

CORRIVEAU, C. (25 novembre, 2011). «Bilan du Plan d'action interministériel en itinérance 2010-2013: Observations et commentaires du Réseau solidarité itinérance du Québec (RSIQ)», <http://www.rapsim.org/docs/ Mémoire \%20-\%20observations \%20et\%20commentaires\%20du\%20 RSIQ.pdf>, consulté le 10 novembre 2012. 
CRANE, M. et A.WARNES (2007). «The outcomes of rehousing older homeless people: a longitudinal study», Ageing \& Society, vol. 27, n 6, p. 891-918. doi : doi : 10.1017/S0144686X07006319

DAVIES, L. (14 avril, 2010). «Libby speaking out against conservatives budget», <http://www.libbydavies.ca/parliament/speech/2010/4/20/libbyspeaking-out-against-conservatives-budget>, consulté le 11 juin 2011.

DENONCOURT, H. M. DÉSILETS, M. PLANTE, M.-C. LAPLANTE, J. et M. CHOQUET (2000). «La pratique outreach auprès des personnes itinérantes souffrant de troubles mentaux graves et persistants: observations, réalités et contraintes », Santé mentale au Québec, vol. 25, n² 2, p. 179-194.

ECHENBERG, $\mathrm{H}$. et H., JENSEN (2008). «L'itinérance au Canada: définitions et recensements», publications de recherche de la bibliothèque du Parlement, <http://www.parl.gc.ca/Content/LOP/ResearchPublications/prb0830f.htm\#fn14>, consulté le 11 juillet 2011.

ESTES, C. L., C. PHILLIPSON et S. BIGGS (2003). Social theory, social policy and ageing: Critical perspectives, England, UK, Open University Press, 200 p.

FALVO, N. (2009). «Homelessness, program responses and an assessment of Toronto's Streets to Homes Program», Toronto, Canada, Canadian Policy Research Networks.

FIRDION, J.-M. (2006). Entre facteurs individuels et facteurs structurels, comment approcher la compréhension de populations en marge des normes sociales? Une approche complexe, thèse de doctorat présentée à I'UQÀM, $352 \mathrm{p}$.

GASCON, M.-H. et I. OLAZABAL (2011). «Le logement social pour aînés à Montréal, un enjeu de taille», Diversité urbaine, vol. 11, n 1, p. 137-142.

GEE, E. et G. GUTMAN (2000). The overselling of population ageing: Apocalyptic demography, intergenerational challenges, and social policy, Oxford University Press, $168 \mathrm{p}$.

GIDDENS, A. (1998). The Third Way: The Renewal of Social Democracy, Cambridge Policy Press, 176 p.

GRENIER, A. (2003). «Unhinging the assumptions within independence: Toward a broader conceptualization of diversity and difference in home care», Canadian Review of Social Policy, vol. 51, p. 29-47.

GRENIER, A. (2007). «Constructions of frailty in the English language, care practice and the lived experience», Ageing \& Society, vol. 27, n 3, p. 425-445. doi : $10.1017 / \mathrm{s} 0144686 \times 06005782$

GRENIER, A. (2012). Transitions and the lifecourse: contested models of 'growing old', Bristol, The Policy Press, $241 \mathrm{p}$.

GRENIER, P. (1996). Still dying for a home, London, Crisis.

HECHT, L. et B. COYLE (2001). «Elderly Homeless : A comparison of older and younger adult emergency shelter seekers in Bakersfield, California», American Behavioral Scientist, vol. 45, n 1, p. 66-79. doi : 10.1177/0002 7640121957024

HOMELESS LINK (août, 2009). «Meaningful activity and older homeless people: A homeless link report on the learning from four projects funded by Help the Aged», Londres, <http://www.homelesshub.ca/Resource/Frame. aspx ?url=http $\% 3 a \% 2$ f\% 2 fhomeless.org.uk\% 2 fsites $\% 2$ fdefault $\% 2 f$ files $\% 2$ fFINAL+Meaningful+Activity+report + .pdf\&id $=53789 \&$ title $=$ Meaningful+Activity+and+Older+Homeless+People\&owner $=121>$, consulté le 9 novembre 2012. 
HULCHANSKI, D. (2009). «Homelessness in Canada: Past, Present, Future», présentation au colloque Growing Home, Housing and Homelessness in Canada, University of Calgary, <http://www.cprn.org/documents/51110_ EN.pdf>, consulté le 11 juillet 2011.

HURTUBISE, R. et M. VATZ LAAROUSSI (2000). «Jeunes dans/de la rue et stratégies de réseaux», dans D. Laberge (dir.), L'errance urbaine, SainteFoy, Éditions MultiMondes, p. 179-192.

JENSON, J. et D. SAINT-MARTIN (2003). «New routes to social cohesion? Citizenship and the social investment state», The Canadian Journal of Sociology / Cahiers canadiens de sociologie, vol. 28, n 1, p. 77-99.

KLODAWSKY, F. (2009). «Home spaces and rights to the city: Thinking social justice for chronically homeless women », Urban Geography, vol. 30, n 6, p. 591-610. doi : 10.2747/0272-3638.30.6.591

KRAMER, J. et J. C. BARKER (1996). «Homelessness among older American Indians, Los Angeles, 1987-1989», Human Organization, vol. 55, n 4, p. 396-408.

KUTZA, E. A. et S.M. KEIGHER (1991). «The elderly "new homeless": An emerging population at risk», Social Work, vol. 36, n 4, p. 288-293.

LABERGE, D., M. POIRIER et R. CHAREST (1998). «Un étranger dans la cité : La présence de l'itinérant et la représentation de l'itinérance», Nouvelles pratiques sociales, vol. $11, \mathrm{n}^{\circ} 1,38-53$.

LAIRD, G. (2007). «Shelter: Homelessness in a growth economy: Canada's 21st century paradox», Calgary, Alberta, Chumir Ethics Foundation.

LAYTON, J. (2008). Homelessness: The making and unmaking of a crisis, Penguin Group Canada.

LENOIR, R. (1974). Les exclus - Un Français sur dix, Paris, Seuil.

LEWIS, J. et R. SURENDER (2004). Welfare state change: Towards a Third Way?, Oxford, UK, Oxford University Press, 248 p.

MCDONALD, L., J. DERGAL et L. CLEGHORN (2007). «Living on the margins», Journal of Gerontological Social Work, vol. 49, n 1 , p. 19-46. doi : 10.1300/ J083v49n01_02

MEANS, R. (2007). «Safe as houses? Ageing in place and vulnerable older people in the UK», Social Policy et Administration, vol. 41, n' 1, p. 65-85. doi : $10.1111 /$ j.1467-9515.2007.00539.x

MERRIEN, F. X. (1996). «État-providence et lutte contre I'exclusion», dans Paugam (dir.), L'exclusion. L'état des savoirs, Paris, La Découverte, p. 417-421.

MINISTÈRE DE L'EMPLOI ET DE LA SOLIDARITÉ SOCIALE (MESS) (2002). «La volonté d'agir, la force de réussir. Stratégie nationale de lutte contre la pauvreté et l'exclusion sociale», Gouvernement du Québec, 20 p.

MINISTÈRE DE LA SANTÉ ET DES SERVICES SOCIAUX (MSSS) (2009). «Plan d'action interministériel en itinérance (2010-2013)», Gouvernement du Québec, 52 p.

MONTPETIT, C. (7 février, 2012). «Vieillir dans la rue - De plus en plus de gens qui ont perdu leur emploi en fin de vie active se retrouvent sans-abri ou très pauvres», journal le Devoir, <http://www.ledevoir.com/societe/ actualites-en-societe/341951/vieillir-dans-la-rue-de-plus-en-plus-degens-qui-ont-perdu-leur-emploi-en-fin-de-vie-active-se-retrouvent-sansabri-ou-tres-pauvres>, consulté le 12 novembre 2012. 
MORRIS, A., B. JUDD et K. KAVANAGH (2005). «Marginality amidst plenty: Pathways into homelessness for older Australians», Australian Journal of Social Issues, vol. 40, n², p. 241-251.

NEGRON-POBLETE, P. (2012). «Des environnements conçus pour répondre aux besoins des aînés. Un défi à relever à plusieurs échelles spatiales», dans P. Negron-Poblete et A.-M. Séguin (dir.), Vieillissement et enjeux d'aménagement. Regards à différents échelles, Québec, Presses de I'Université de Québec, p. 1-9.

OLD BREWERY MISSION (OBM). (2009-2010). «Documents internes: Compilation statistique des programme d'accompagnements», Montréal, Québec, Old Brewery Mission.

OFFICE MUNICIPAL D'HABITATION DE MONTRÉAL (OMHM) (2011). «Waiting lists and studying and ranking applications », <http://www.omhm.qc.ca/ en/waiting-lists-and-studying-and-ranking-applications>, consulté le 31 octobre 2011.

OUDSHOORN, A. (2011).Client-provider relationships in a community health clinic for people experiencing homelessness: A critical ethnography, thèse de doctorat présentée à la University of Western Ontario, 352 p.

PANNELL, J., R. MEANS et H. MORBEY (2002). Surviving at the margins: Older homeless people and the organizations that support them, London, Help the Aged.

PANNELL, J. (2005). «Extra Care Housing Models and Older Homeless People», London, UK Department of Health, Housing Learning and Improvement Network, <http://www.olderhomelessness.org.uk/documents/extra_carefact sheet16.pdf>, consulté le 10 novembre 2012.

PARAZELLI, M. (2002). La rue attractive, Québec, Presses de I'Université du Québec, $382 \mathrm{p}$.

PARNABY, P. (2003). «Disaster through dirty windshields law, order and Toronto's squeegee kids», The Canadian Journal of Sociology / Cahiers canadiens de sociologie, vol. 28, n 3, p. 281-307.

PARSELL, C. et M. PARSELL (2012). «Homelessness as a choice», Housing, Theory and Society, vol. 49, n 4, p. 420-434.

PAUGAM, S. (1996). L'exclusion. L'état des savoirs, Paris, Éditions La Découverte, $583 \mathrm{p}$.

POIRIER, M. et J. GAGNÉ (1988). «Formes de I'appauvrissement et insertion sociale des jeunes adultes psychiatrisés», Santé mentale au Québec, vol. $13, \mathrm{n}^{\circ} 1$, p. $132-143$.

POIRIER, M., V. LUSSIER, R. LETENDRE, P. MICHAUD, M. MORVAL, S. GILBERT et A. PELLETIER (1999). «Relations et représentations interpersonnelles de jeunes adultes itinérants», Montréal, Groupe de recherche sur l'itinérance des jeunes adultes, rapport de recherche soumis au Conseil québécois de la recherche sociale, $179 \mathrm{p}$.

POTTER, J. et autres (2005). Ottawa's 2006-2008 Community Action Plan on Homelessness to create «A City Without Homelessness», Ottawa, Community Capacity Building Team, 60 p.

QUIRION, B. et A. DI GENNARO (2000). «L'inflation des cas de problématiques multiples: le symptôme des disjonction entre la logique institutionnelle et la phénoménologie des besoins », dans D. Laberge (dir.), L'errance urbaine, Sainte-Foy, Éditions MultiMondes, p. 329-346. 
RAPSIM (2010). «L'itinérance à la hausse: Raison pour agir!», <http://www. rapsim.org $/ \mathrm{fr} /$ default.aspx ?sortcode $=1.12 \&$ id_article $=543 \&$ starting $=\&$ ending=>, consulté le 17 août 2010.

RAPSIM (25 novembre, 2011). «L'action du gouvernement du Québec en itinérance depuis 2009: Un bilan qui rappelle l'urgence d'instaurer une Politique en itinérance», <http://www.rapsim.org/docs/Bilan_RAPSIM_ action_en_itinerance_pour_CSSS-25nov2011.pdf>, consulté le 12 décembre 2012.

RAPSIM (novembre, 2012). «Enfin une politique itinérance!», <http://www. rapsim.org/fr/default.aspx ?sortcode $=1$.0\&id_article $=660 \&$ starting $=$ \&ending $=>$, consulté le 2 novembre 2012.

RESSOURCES HUMAINES ET DÉVELOPPEMENT DES COMPÉTENCES CANADA (RHDCC) (2011). «La Stratégie des partenariats de lutte contre l'itinérance (SPLI)», <http://www.rhdcc.gc.ca/fra/sans_abri/apropos_nous/index.shtml>, consulté le 11 juillet, 2011.

REVENU QUÉBEC (2011). «Programme Allocation-logement», <http://www. revenuquebec.ca/fr/citoyen/credits/programmes/programmes_Allocationlogement.aspx>, consulté le 11 juillet 2011.

RÉSEAU SOLIDARITÉ ITINÉRANCE DU QUÉBEC (RSIQ) (25 novembre, 2011). «Bilan du Plan d'action interministériel en itinérance (2010-2013): Observations et commentaires du Réseau solidarité itinérance du Québec (RSIQ)», <http://www.rapsim.org/docs/Mémoire\%20\%20observations\%20 et\%20commentaires\%20du\%20RSIQ.pdf>, consulté le $1^{\text {er }}$ novembre 2012.

ROWE, J. W. et R.L. KAHN (1987). «Human aging: usual and successful», Science, vol. 237, p. 143-149.

ROY, S. (1995). «L'itinérance: forme exemplaire de l'exclusion sociale?», Lien social et politiques, vol. 34, p. 73-80.

ROY, S., D. LABERGE, P. MORIN et M. ROZIER (2002). «Itinérance, détresse psychologique et prévention», dans G. Godin, J. Levy et G. Trottier (dir.), Vulnérabilités et prévention VIH/SIDA. Enjeux contemporains, Québec, Les Presses de I'Université Laval, $344 \mathrm{p}$.

ROY, S. et R. HURTUBISE (2007). L'itinérance en questions, Québec, Presses de I'Université du Québec, 398 p.

ROY, S. et R. HURTUBISE (2008). «La lutte à I'itinérance. Une responsabilité collective qui nécessite un leadership de l'État», mémoire présenté à la commission parlementaire sur l'itinérance, Québec, Collectif de recherche sur l'itinérance, la pauvreté et l'exclusion sociale.

RSIQ (2009). «The Federal Government must promptly step up the struggle against homelessness 》, <http://www.rapsim.org/docs/RSIQ_comm25no09 _ang.pdf>, consulté le 8 octobre, 2012.

SANTÉ QUÉBEC (novembre, 2008). «Dénombrement de la clientèle itinérante dans les centres d'hébergement, les soupes populaires et les centres de jour des villes de Montréal et de Québec 1996-97», sous la direction de Louise Fournier et al.

SCHARF, T., C. PHILLIPSON et A.E. SMITH (2005). «Multiple exclusion and quality of life amongst excluded older people in disadvantaged neighbourhoods», London, Office of the Deputy Prime Minister, Social Exclusion Unit. 
SERVICE CANADA (2011). «Programme de la sécurité de la vieillesse (SV)», <http://www.servicecanada.gc.ca/fra/psr/sv/svtabmat.shtml>, consulté le 12 septembre 2011.

SOCIAL EXCLUSION UNIT (2004). «Breaking the cycle: Summary, taking stock of progress and priorities for the future», London, Office of the Deputy Prime Minister.

SILVER, H. (1994). «Social exclusion and social solidarity: three paradigms», International Labour Review, vol. 133, p. 531-578.

SNYDER, L. (2006). «Workfare: Ten years of pickin' on the poor», dans A. Westhues (dir.), Canadian social policy: Issues and perspectives (4e éd.), p. 309-331), Waterloo, ON, Wilfrid Laurier University Press.

UK COALITION ON OLDER HOMELESSNESS (2007). <http://www. homelesshub. ca/Library/The-Uk-Coalition-on-Older-Homelessness-36734.aspx>, consulté le 12 novembre 2011.

TSEMBERIS, S. et R.F. EISENBERG (2000). «Pathways to housing: Supported housing for street-dwelling homeless individuals with psychiatric disabilities », Psychiatric Services, vol. 51, n 4, p. 487-493.

VILLE DE MONTRÉAL (2011). «Agir résolument pour contrer l'itinérance: Plan d'action en itinérance», <http://ocpm.qc.ca/sites/default/files/pdf/ P62/5g.pdf>, consulté le 11 juillet 2011.

VRANKEN, J. (2002). «No social cohesion without social exclusion ? »,Università di Urbino, Italie, <www.shakti.uniurb.it/eurex/syllabus/lecture4/ Eurex4_ Vranken.pdf>, consulté le 25 février 2008.

WALKER, A. (1982). «Dependency and old age», Social Policy \& Administration, vol. 16, n² 2, p. 115-135. doi : 10.1111/j.1467-9515.1982.tb00164.x

WORLD HEALTH ORGANIZATION (WHO) (2002). «Active ageing a policy framework», Geneva, $\mathrm{CH}$, WHO's Ageing and Lifecourse Programme.

\section{NOTES}

1 Selon Rowe et Kahn (1987) le vieillissement réussi se caractérise par un faible taux de maladies et d'incapacités ou un faible risque d'en développer, un bon fonctionnement physique et cognitif, de même qu'une vie sociale active. Voir Grenier (2012) pour une présentation de cette approche d'analyse du discours et une critique de l'imposition de ces discours à des groupes de personnes âgées marginalisées.

2 Le paradigme de l'investissement social a influencé les programmes d'aide sociale en associant les prestations à la recherche d'emploi (Workfare). Voir Snyder (2006) sur I'approche Workfare au Canada.

3 Voir Alberta Secretariat for Action On Homelessness (2008), City Of Vancouver (2005), et Potter, J. et autres (2005).

4 Pour une discussion sur la dépendance et le vieillissement voir Alan Walker (1982).

5 On remarque que la Stratégie des partenariats de lutte contre l'itinérance du gouvernement canadien encourage explicitement I'approche du Housing First. 
6 Terme tiré de I'anglais qui désigne au Québec les jeunes adultes itinérants qui se positionnent aux feux de circulation avec leur raclette pour laver les parebrises et ainsi obtenir de l'argent des automobilistes.

7 Il est important de noter que les montants annuels accordés (20 millions \$ par an au Québec, dont 7,8 millions \$ à Montréal) à ce programme sont gelés depuis dix ans, malgré l'inflation et la hausse constante du nombre des personnes itinérantes. Les coûts des projets immobiliers ont notamment presque triplé depuis 1999 (Communiqué RAPSIM, mai 2011).

8 Au 16 juillet 2012, 2047 projets avaient été acceptés et avaient reçu plus de 693 millions \$ en subvention de la SPLI afin de prévenir ou réduire I'itinérance au Canada (voir < http://actionplan.gc.ca/fr/fiche-d-information/ la-strat-gie-des-partenariats-de-lutte-contre-l-itin-rance $>$ )

9 Bizarrement, le texte de présentation en français de la Stratégie est complètement différent et on n'y trouve aucune référence à l'étiologie de l'itinérance.

10 Le RAPSIM, créé depuis 1974, regroupe plus de 90 organismes intervenant directement et indirectement auprès des personnes en situation d'itinérance et à risque à Montréal. Sa mission est la défense des droits des personnes itinérantes et des intérêts de ses groupes membres, $<$ www.rapsim.org $>$.

11 Le RSIQ représente plus de 200 organismes au Québec qui interviennent auprès de milliers de personnes en situation ou à risque d'itinérance.

12 La gérontologie critique s'inscrit dans la tradition postmoderne et poststructuraliste dont l'objectif épistémologique est de déconstruire les discours, les institutions et les représentations qui dominent en gérontologie. Elle cherche à contester la gérontologie classique et à amener les gérontologues à remettre en question les présupposés ou les biais qui orientent leur pratique. 